\title{
Digital Timing Error Calibration of Time-Interleaved ADC with Low Sample Rate
}

\author{
Marko Neitola \\ ITEE/CAS Group \\ University of Oulu, Finland \\ ORCID iD: 0000-0002-0213-3793
}

\begin{abstract}
This work suggests a novel procedure of calibrating timing mismatch of time-interleaved Analog-to-Digital conversion. Here, the timing mismatch calibration and compensation are both performed digitally. The proposed method is simple and it is able to find timing errors below the Nyquist rate of the individual analog-to-digital converter. Moreover, the method can be easily modified for digital gain error calibration.
\end{abstract}

Index Terms-time-interleaving, ADC, mismatch calibration

\section{INTRODUCTION}

The purpose of time-interleaving technique in analog-todigital (A/D) conversion is to lower the sampling rate in A/D interface. Time-interleaved architectures exploit parallelism to enable sampling rates that are not achievable (or power inefficient) in single-channel A/D converters [1]. By interleaving the data into $\mathrm{N}$ channels or paths, the conversion is performed by $\mathrm{N}$ individual $\mathrm{A} / \mathrm{D}$ converters operating at $f_{s} / \mathrm{N}$, $f_{s}$ being the data rate of the combined time-interleaved $\mathrm{A} / \mathrm{D}$ converter (ADC) output. Well-known drawbacks of a timeinterleaved ADC (TI-ADC) are caused by the mismatches between individual paths.

The main mismatches in TI-ADC are gain error, offset error and timing error. Gain and offset errors correspond to mismatches between ADCs and timing error refers to sampling time skew i.e. small aperture delay mismatches between paths.

This work focuses on calibrating timing mismatches in from the digital output of interleaved data. The correction (error compensation) will be performed to the digital data. The proposed method falls in the category of blind background calibration i.e. no offline training stimulus (pilot input) will be used.

In [2], Vogel and Johansson reviewed several publications on timing mismatch calibration/correction and concluded that the goal of timing mismatch correction method for in TI-ADC is to find an accurate, power-aware method, which only needs a slight oversampling and which can be easily adapted to changing timing mismatches. This work suggest a method that does not require oversampling of the individual Sub-ADC.

In [3], Jamal et al. proposed adaptive timing error calibration for a 2-path (2-channel) TI-ADC. Their strategy was to upsample both channels by $\mathrm{N}$ (number of paths) in order to to synchronize the paths that for the adaptive feedback loop. The learning data from adaptive filtering block was obtained from spurious tone, limiting the Sub-ADC data bandwidth to be less than the Nyquist band, as in e.g. [4], [5]. In [6], Law et al. expanded the idea in [3] to a four-channel TI-ADC.

The method proposed in this work uses the idea of channel synchronization as in [3], [6], but without upsampling and with a different adaptation logic for the timing error mitigation. The suggested digital calibration scheme is capable of calibrating timing errors even from above Nyquist bandwidth of SubADCs.

Section II discusses the oversampling ratio and demonstrates overlapping spurious tone clusters (i.e. birdies) in TI-ADC, due to low data rate. Section III introduces the proposed calibration method and the optimal parameters are discussed in Section IV. Section IV also discusses the performance of the proposed calibration method.

As the focus in this work is at the timing mismatch calibration, it is noteworthy that the proposed digital calibration method is insensitive to the gain and offset errors. This means that the gain and offset error calibration can be done in parallel with timing mismatch calibration. The proposed method can be modified to calibrate gain mismatch and this will be presented in Section V.

\section{Sub-ADC Oversampling RATIO}

The spurious tones due to TI-ADC mismatches (a.k.a. birdies ) were analyzed by Kurosawa et al. in [7]. Gain, offset and timing errors between Sub-ADCs cause spurious images and spikes centered at $f_{c_{\text {spur }}}$ according to the number of paths, as shown in (1).

$$
f_{c_{\text {spur }}}=k \cdot f_{s} / N, \text { where } k=1,2, \cdots, N
$$

The characteristic birdies due to time-interleaving are demonstrated in Fig. 1, where we have a 12-bit 7-path TI-ADC with a multi-tone (11 tones) as a stimulus. Figure 1 depicts three different mismatch mechanisms: offset (Fig. 1a), gain (Fig. 1b) and timing mismatch (Fig. 1c). The oversampling ratio (OSR) for Sub-ADCs in Fig. 1 is two, i.e. the data rate of each Sub-ADC is twice the Nyquist rate.

If the sampling rate of Sub-ADCs is less than the Nyquist rate, the spurious tone clusters in the TI-ADC output will alias and tend to overlap with each other and with the fundamental. If we reduce the OSR from 2 to 4/7, the data in the Sub-ADC is undersampled, see Fig. 2. 


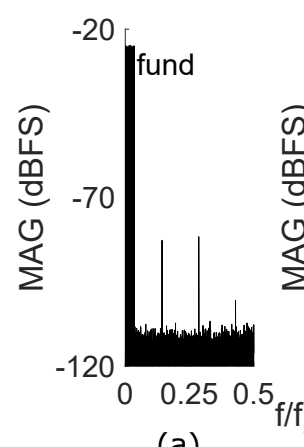

(a)

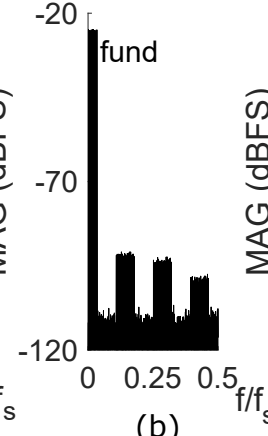

(b)

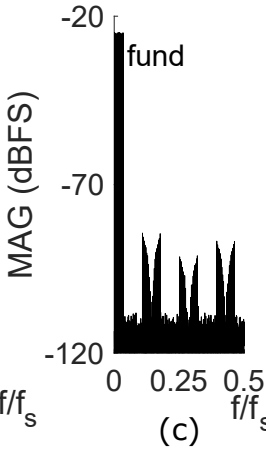

Fig. 1. 12-bit TI-ADC output with $\mathrm{N}=7$ and $\mathrm{OSR}=2$ and with a) offset, b) gain and c) timing mismatch.
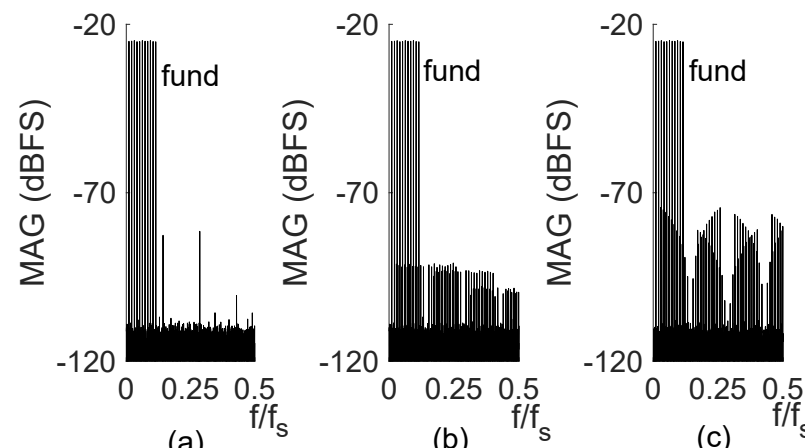

Fig. 2. 12-bit TI-ADC output with $\mathrm{N}=7$ and $\mathrm{OSR}=4 / 7$ and with a) offset, b) gain and c) timing mismatch.

The combined TI-ADC output sampling rate $\left(f_{s}\right)$ is $\mathrm{N}$-fold compared to a Sub-ADC, which means that the TI-ADC output sampling rate shown in Fig. 1 and Fig. 2 will also be Nfold. To avoid confusion, the term OSR will be here tied to the Sub-ADC. The N-fold TI-ADC oversampling ratio will be denoted as samples-per-symbol (sps). The connection between sps, OSR and Sub-ADC bandwidth (BW) are shown in (2) and (3).

$$
\begin{aligned}
\mathrm{OSR} & =\mathrm{sps} / N \\
\mathrm{BW} & =\frac{f_{s} / N}{2 \cdot \mathrm{OSR}}=\frac{f_{s}}{2 \cdot \mathrm{sps}}
\end{aligned}
$$

In the art of digital mismatch calibration of an individual channel, low oversampling ratio poses challenges. If part of the spectrum is dedicated as a spurious tone measurement, it is imperative that the measurement band has no fundamental and/or aliased fundamental. Digital fractional delay filters (baseband FIR or IIR) may also be problematic as they are known to be less accurate near $f_{s} / 2$ [8].

\section{Digital Timing ERror CAlibration}

The structure of the proposed background calibration scheme is shown in Fig. 3. Thick data-flow arrows in Fig. 3 represent two or more data paths in parallel. Input data are quantized outputs of $\mathrm{N}$ paths operating at $f_{s} / N$.

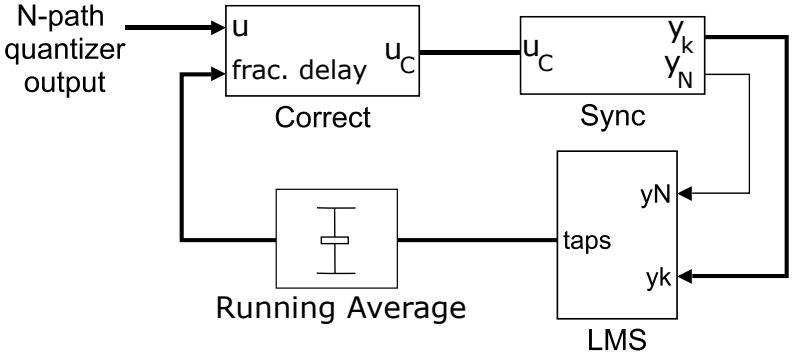

Fig. 3. The Simulink-model of the proposed digital background calibration scheme.

The Correct block contains a fractional delay filter that delay or advance the data according to the input delay (which is initially all zeros). The fractional delay inputs in the Correct block originate from adaptive LMS block. These delays change by sampling instant until the delays converge.

The Sync block synchronizes the timing of all $\mathrm{N}$ paths by fixed fractional delay filtering. Basically, one path will be declared as a path with no aperture delay mismatch. The timing mismatches in other paths will be adapted until convergence. The consequence of declaring no timing error for one path is that there will be an additional aperture delay common to all delay estimates. This added aperture delay will only contribute to the overall delay, not the birdies.

The adaptation in Fig. 3 has properties that will cause fluctuation in LMS tap convergence. The fluctuation will be smoothed by a simple running average filtering for all N-1 LMS taps. The taps are negative timing mismatch estimates to be used in the compensation (Correct block).

\section{A. Channel Synchronization}

The Sync block in Fig. 3 synchronizes the data path delays between $\mathrm{N}$ paths at the Sub-ADC sample rate of $f_{s} / N$. The Sync block is detailed in Fig. 4, where the data path goes through fractional delay filters with fixed delays.

The reference point of the synchronization will be taken from the most delayed data path. Assuming the most delayed data is $y_{N}[n]$, the delay of $y_{k}[n]$ compared with $y_{N}[n]$ is:

$$
d_{k}=\frac{N-k}{N}, k=1,2, \cdots N
$$

The synchronization of the data paths $y_{1}[n] \cdots y_{N-1}[n]$ cancels the delays in (4). The cancellation will be performed by fixed-delay fractional-delay FIR filters. Choosing a fractional delay filtering prototype function has many alternatives [8], which are discussed in Section IV.

As mentioned in the Introduction, [3], [6] had similar synchronization idea but at full sample rate of $f_{s}$, which results in integer delay synchronization.

\section{B. Adaptation}

After synchronization, [3], [6] suggested adaptive filtering for the timing error detection. Their strategy was to isolate the birdy responsible to timing mismatch and use it as the noise reference. In this work, the calibration is based on correlating 


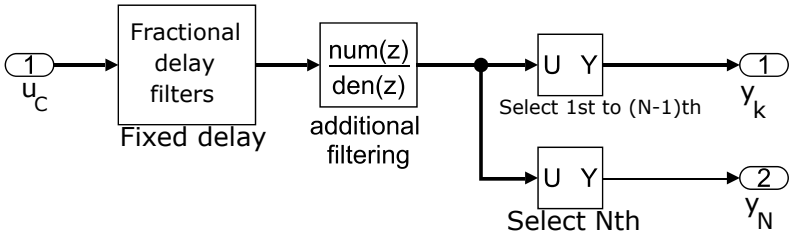

Fig. 4. The Synchronization (Sync) block.

the baseband fundamental between a reference path and the other paths.

By not using spur-related noise reference, we are able to experiment an additional lowpass filter after the fixed fractional delays in Fig. 4. The additional lowpass-filter is the key on enabling timing error mitigation of subsampled SubADCs below Nyquist rate: the in-band birdies can be filtered by some extent. Proper cutoff frequency for the lowpass-filter depends on the oversampling ratio of the Sub-ADC. The cutoff and the conditions under which the lowpass filter is beneficial are discussed in Subsection IV-B.

The adaptive logic in Fig. 5 is based on the simple and robust Least Mean Squares filtering [9]. The upper input to the multiplier in Fig. 5 represents the error whose mean squared error will be minimized. This input is the data $-y_{k}(n)$ filtered by $\left(1-z^{-2}\right)$. Such filtering adds a notch at dc and $f_{s} / 2$, which cancels the fractional delay output at its most errorprone frequency range i.e. near $f s / 2$. This means that the fractional filtering errors at $f_{s} / 2$ will not hinder the calibration.

The notch at dc due to $\left(1-z^{-2}\right)$ filtering cannot be avoided and it will contribute to LMS tap fluctuation, whose frequency is dependent on the calibration loop delay. As mentioned, the running average filtering in Fig. 3 will cancel the fluctuation.

The lower multiplicand in Fig. 5 is the reference data $y_{N}(n)$, which will be delayed by one sample. This will synchronize the multiplicands.

By using a behavioral model, the choice of LMS correction can be tested without an adaptation feedback. Suppose you have simulated long arrays (length L) of synchronized path outputs (5). The matrix inversion in (6) with the reference array results in two coefficients, whose subtraction (7) results in (negative) approximated mismatch. The result correlates with the actual timing mismatch.

$$
\begin{aligned}
\mathbf{y}_{\mathbf{k}} & =\left[y_{k}(1), y_{k}(2), \cdots y_{k}(L)\right]^{T} \\
C_{k} & =\left[-\mathbf{y}_{\mathbf{k}}(\mathbf{n}), \mathbf{y}_{\mathbf{k}}(n-2)\right]^{-1} \cdot \mathbf{y}_{N}(n-1) \\
-\hat{d}_{k} & =C_{k}(1)-C_{k}(2)
\end{aligned}
$$

As known in the theory of LMS algorithm, the adaptation constant $\mathrm{mu}(=\mu)$ in Fig. 5 dictates the speed and accuracy of the learning curve. In theory, $\lambda_{\max }$ in (8) is the largest eigenvalue of the autocorrelation matrix of the input data $y_{N}$ [10]. The additional filtering of the data also contributes to the maximal (and optimal) value if $\mu$. The optimization of $\mu$ is discussed in Section IV.

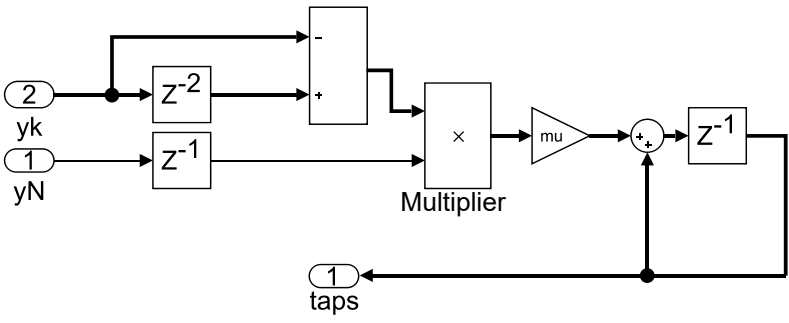

Fig. 5. The LMS block.

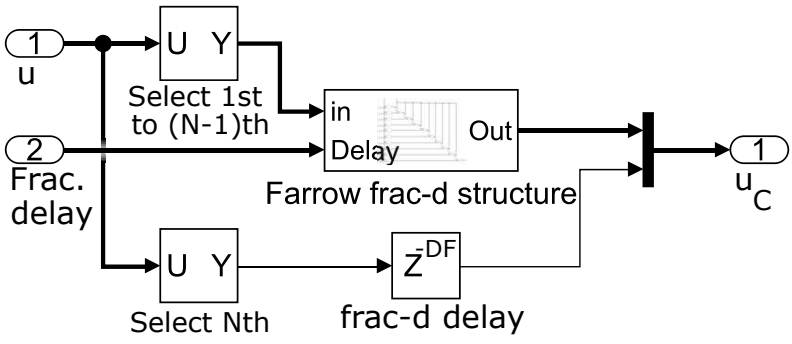

Fig. 6. The Correct block.

$$
0<\mu<2 / \lambda_{\max }
$$

\section{Digital Timing Error Compensation}

The LMS weights or taps in Fig. 5 are the negative timing errors that are fed into the Correct block shown in Fig. 3. A closer look at the Correct block is shown in Fig. 6. The taps are fed into a fractional delay Farrow structure [11], which performs the delay for the channels from 1 to $\mathrm{N}-1$. The Farrow structure enables delay filtering by varying fractional delays.

As the $\mathrm{N}^{t h}$ path is the reference, it will be delayed by the overall delay of the fractional delay filters. For a FIR type fractional delay with the order of $N_{F I R}$, the integer delay DF will be as in (9) [8].

$$
D F=\left\lfloor N_{F I R} / 2\right\rfloor
$$

\section{OPTIMIZATION}

As mentioned in the Introduction, a mismatch calibration and compensation method should be power-aware. For the calibration method suggested here, the design trade-offs (complexity and power consumption vs. convergence and performance) would concentrate on the fractional delay filter (continuously variable Farrow structure [11]) and the additional lowpass filter.

However, this work does not concentrate on a single fixed system so the focus is on performance optimization. The aim in this Section is to demonstrate that the proposed method is functional with any number of paths and different oversampling ratios. 


\section{A. Fractional Delay Filtering}

The synchronization and correction blocks in Figures 4 and 6 both contain fractional delay filtering. There are many prototype FIRs to choose from, and the Author favored these candidates:

- 3-parameter optimal offset window [12]

- Direct Least Squared Integral Error FIR Design [13]

The above prototypes were found suitable for both fixed delay filtering (Fig. 4) and variable-delay Farrow structure [11] (Fig. 6). The Farrow structure [11] is a collection of polynomially fitted filter coefficient arrays from which the proper filter coefficients are evaluated according to the delay input.

To obtain the Farrow polynomial coefficients requires generating a sets of filter coefficients based on different delay values. In the optimizations, the filter order $N_{F I R}$ was 9 , the Farrow polynomial order was also 9. The Farrow polynomial (from which the filter coefficients are extracted), was constructed by using an extensive range of 15 different delays.

For the correction block in Fig. 6, the chosen fractional delay FIR prototype is the 3-parameter optimal offset window [12]. For the fixed delays in the synchronization in Fig. 4, General LS design prototype [13] was used.

The fractional delay parameters, the size of the Farrow structure and prototypes were chosen by testing fractionally delayed multi-tone magnitude and phase errors.

\section{B. LMS and Additional Lowpass Filtering}

For the LMS loop in Fig. 5, the maximal LMS step size $\mu$ in (8) will depend on both stimulus and filtering: The additional lowpass filtering in Fig. 4 will increase the upper limit of $\mu$.

Through the behavioral simulation sweeps (presented in Subsection IV-C), the lowpass filtering was found beneficial as the spurs and the aliased fundamental are filtered out. For low OSR $(0.5<O S R \leq 2)$, the additional lowpass filter is crucial as the spurs and aliased fundamental distort the adaptation.

LMS optimization is a multi-dimensional problem with the presence of the additional lowpass filter. The LMS learning curve should be optimized for maximal convergence speed and accuracy for given lowpass filter cutoff frequency $f_{c}$ and $\mu$. Moreover, the type (FIR, IIR), the order, cutoff and the prototype of the lowpass filter needs to be optimized.

The LMS optimization routine can be divided into two parts. In the first part, we'll optimize the lowpass filter order and cutoff while the LMS step-size is fixed to a small $\mu$ $\left(\mu<<\mu_{\max }\right)$. The figure of merit in the first part is the Pearson's correlation coefficient [14] R (10) between the arrays of true and estimated timing errors. Pearson's correlation coefficient (10) is quite insensitive to the slope error between the arrays of true and estimated timing errors. The cutoff is optimized by (10) with the optimization error criterion of 1-R. The optimized filter orders varied between 4 to 5 for the IIR and 13 to 30 for the FIR filter.

In the second part, $\mu$ is optimized for minimal variance between the arrays of true and estimated aperture delays per path. The true timing mismatches are, of course, taken from a behavioral model. The maximal value of $\mu$ in (8) is naturally dependent on the type of stimulus.

In the sweeps discussed below and in Section IV-C, a 17-tone stimulus was used with maximal value of $90 \%$ of full scale. The optimization results for $\mu$ reported below will therefore be suited with stimuli with high peak-to-average power.

$$
R=\frac{\operatorname{cov}(\mathbf{d}, \hat{\mathbf{d}})}{\sigma(\mathbf{d}) \sigma(\hat{\mathbf{d}})}
$$

The optimized cutoff frequencies and the LMS step size $\mu$ for the case of FIR filter are shown in Fig. 7. For IIR filter, the graphs were very similar and are not depicted here. The initial guess for the cutoff frequency (shown in Fig. 7a) is defined in (11) and (12). Not that the optimized $f_{c}$ are very close to the initial values, especially for OSR $>1$.

$$
\begin{aligned}
& f_{c}=\mathrm{BW} / 2, \text { OSR } \geq 1 \\
& f_{c}=(1-\mathrm{BW}) / 2,0.5<\mathrm{OSR}<1
\end{aligned}
$$

At certain parameters, the LMS step size versus OSR is eligible for fitting. From Fig. $7 b$, the step size $\mu$ is quite accurately in-line with the approximation in (13) for OSR $\geq 1$. From (2), (3), (11) and (13), the linearized relationship between $f_{c}$ and $\mu$ can be deducted, see (14).

$$
\begin{aligned}
\mu & \approx 0.188 / \mathrm{OSR}, \mathrm{OSR} \geq 1 \\
\rightarrow \mu & \approx 0.75 \cdot f_{c}, \mathrm{OSR} \geq 1
\end{aligned}
$$

For $0.5<\mathrm{OSR} \leq 1$, the optimization will get more challenging and a polynomial dependencies between $\mu, f_{c}$ and OSR are harder to obtain. For OSR $\in] 0.6,1[$ we can fit a coarse approximation (15), but as we approach the $\mathrm{OSR}=0.5$ boundary, $\mu$ will increase rapidly.

It should be emphasized here, that the sweeps performed for this work included fixed fractional delay filters and two lowpass filter prototypes. For a specific environment with fixed sps and $\mathrm{N}$, more alternatives should be studied.

$$
\mu \approx-0.18 \cdot \text { OSR }+0.26, \text { OSR } \in] 0.6,1[
$$

The practical limits for minimum OSR and maximum bandwidth versus number of paths $\mathrm{N}$ are presented in (17) and (18), respectively. These limits are the based on the behavioral simulations. Equation (17) clearly states that the calibration method presented here is limited to OSR $>0.5$ (Sub-ADC oversampling ratio). At $\mathrm{OSR}=0.5$, the aliased fundamental fills the frequency band at $f_{s} / \mathrm{N}$.

$$
\begin{aligned}
\text { sps } & \geq\lfloor(N) / 2\rfloor+1 \\
\Rightarrow O S R & \geq \frac{\lfloor(N) / 2\rfloor+1}{N} \\
\Rightarrow B W & \leq N \cdot \frac{f_{s}}{2 \cdot(\lfloor(N) / 2\rfloor+1)}
\end{aligned}
$$



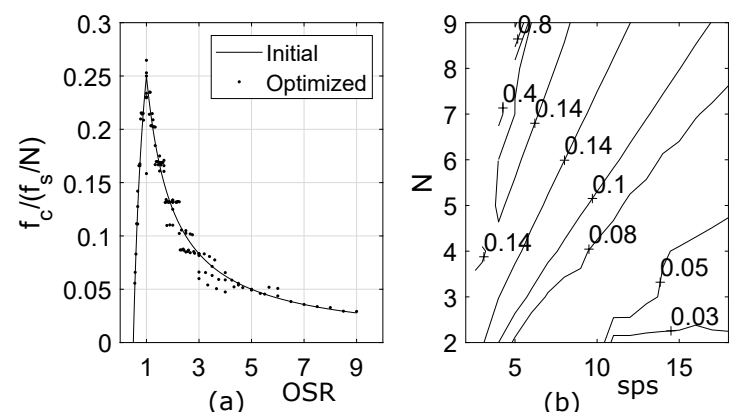

Fig. 7. Optimized parameters for the sweep with FIR filter: a) Cutoff $f_{c}$ (Normalized by $f_{s} / N$ ) and b) $\mu$ versus sps and $\mathrm{N}$.

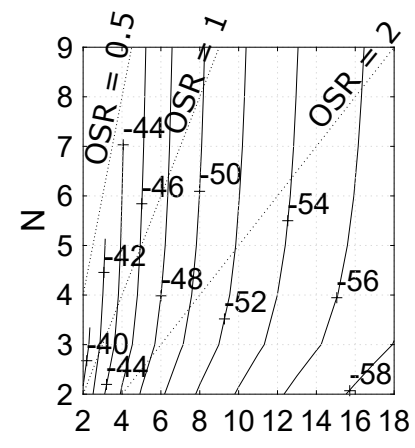

(a) $\mathrm{sps}$

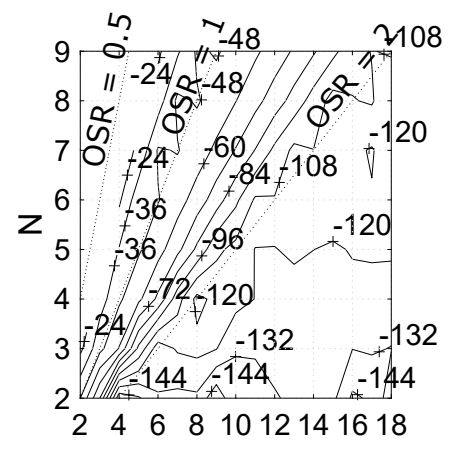

(b) $\mathrm{sps}$
Fig. 8. Timing error distortion power (dBFS) for an N-path ADC with 17-tone input a) without timing mismatch correction, b) with correction, but without additional filtering.

\section{Optimization Sweep Results}

In Fig. 8, the number of paths and sps are swept and the timing error related distortion power is shown as a contour plot. Here, the aperture delay mismatches are randomly generated with fixed standard deviation of $0.1 / f_{s}$.

Without calibration, the distortion power contours are shown in Fig. 8a. Considering quantizer noise floor of around 73 $\mathrm{dB}$ (12-bit TIADC), the noise power due to timing mismatch dominates. In Fig. 8b, the calibration has been used but without any additional lowpass filtering. The calibrations seems to work selectively: the noise floor drops for OSR $\leq 2$.

Note that the results in Fig. $8 \mathrm{a}$ and $8 \mathrm{~b}$ do not include quantizer noise but only the distortion power from the timing errors.

In Fig. 9, the additional filtering is active. In cases of FIR and IIR filtering, the calibration is able to find the timing mismatches quite accurately. As shown in Fig. 9 (both subplots), the timing error induced noise floor is below the quantizer noise floor (around $73 \mathrm{~dB}$ for 12-bit TI-ADC).

At various OSRs swept, the convergence varied roughly from a few ksamples to a 20 ksamples. As mentioned in Subsection IV-B, many cases are covered ( $\mathrm{N}$ and sps were swept). For a fixed environment, optimization e.g. choosing filter prototypes should be more thorough to improve convergence.

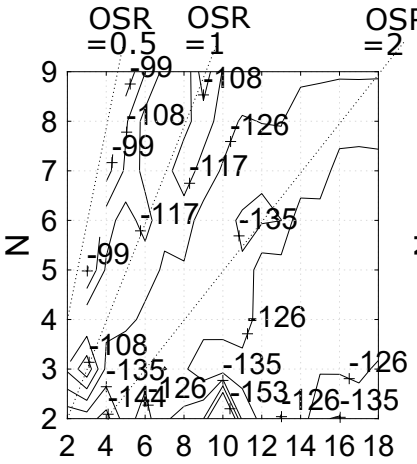

(a) $\mathrm{sps}$

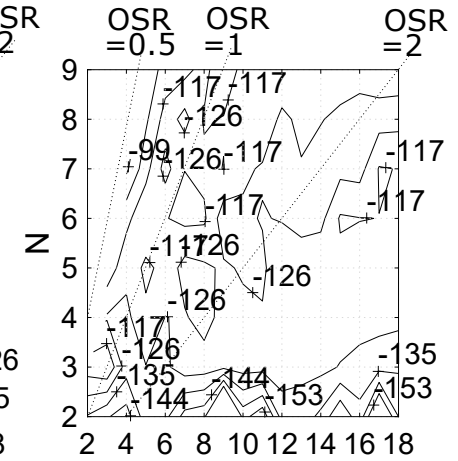

(b) $\mathrm{sps}$
Fig. 9. Timing error distortion power (dBFS) for an N-path ADC with 17tone input with timing mismatch correction and with a) FIR filtering and b) IIR filtering.
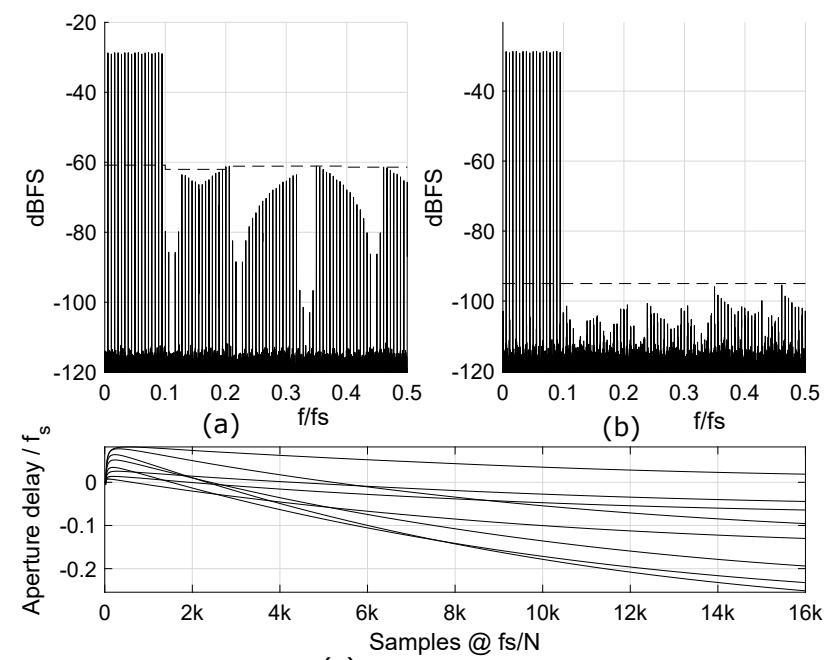

(c)

Fig. 10. 12-bit TI-ADC with 17-tone stimulus, $\{\mathrm{N}, \mathrm{OSR}\}=\{9,5 / 9\}$ : a) without correction, b) with correction and c) LMS tap convergence for the latter.

Figure 10 represents an example with extremely low oversampling ratio $(\mathrm{OSR}=5 / 9)$. Figures $10 \mathrm{a}$ and $10 \mathrm{~b}$ demonstrate a 12-bit 9-path TI-ADC system with 17-tone stimulus at OSR of 5/9: before and after correction. The corrected timing errors reduce the distortion power for about $35 \mathrm{~dB}$. The error convergence for the case of Fig. 10b is depicted in Fig. 10c. The array of estimated errors were obtained at around 16 ksamples (at $f_{s} / N, \mathrm{~N}=9$ ).

From the sweeps, it can be concluded that the suggested timing error calibration method is successful on measuring timing errors even at $\mathrm{OSR} \leq 1$. As the focus here was on calibration, a few remarks on actual correction should be discussed.

If the corrected TI-ADC output is taken from the output of the Correct block in Fig. 3, the fractional delay filter properties may have a negative impact on the performance. As mentioned, the fractional delay filters are typically the least ideal at half the sampling rate $\left(f_{s} /(2 N)\right)$. This drawback 


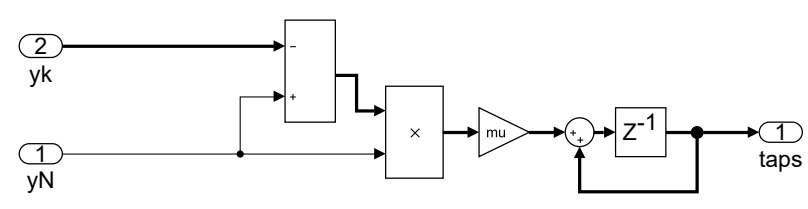

Fig. 11. LMS block for the gain error calibration.

was here avoided by the LMS error reference design (Subsect. IV-B). However, if the corrected TI-ADC output $u_{C}(n)$ (Fig. $3)$ is combined into rate $\left(f_{s}\right)$, the output may contain elevated spurious tone levels for $\mathrm{OSR} \leq 1$.

In the performance sweeps presented above, the fractional delay filter imperfections were omitted by subtracting the calibrated timing mismatch estimate from the real timing error i.e. the fractional delay filters did not affect the performance results.

In the case of a quadrature baseband TI-ADC, the fractional delay filter related distortion would be avoided by converting LMS taps for in-phase (I) and quadrature-phase (Q) into a correcting I/Q phase shift.

\section{Gain AND OfFSet ERror Calibration}

The timing error calibration scheme is insensitive to gain and offset errors. This means that the calibration of such errors can be done in parallel with aperture delay calibration. Even though the main focus in this work is on timing mismatch calibration, the gain and offset errors are addressed in this Section.

The LMS block for the gain error calibration is shown in Fig. 11, where the error data is the subtraction between $y_{N}(n)$ and $y_{k}(n)$. The taps in Fig. 11 correspond to negative gain errors $\hat{e}_{\text {gain }}$, which will be added to cancel the actual gaing error in the main model shown in Fig. 12. Here, gain error $e_{\text {gain }_{k}}$ is modeled as in (19).

$$
u_{k}(n) \rightarrow u_{k}(n) \cdot\left(1+e_{\text {gain }_{k}}\right)
$$

The Sync block in Fig. 12 is copied from Fig. 4. As in the timing mismatch calibration, the zero-error reference in the gain error calibration will be taken from the $\mathrm{N}^{t h}$ path. This means that the all calibrated gain errors will contain a bias error not contributing to spurious tones. An example of gain error calibration is shown in Fig. 13, where the parameter $\mu$ and the lowpass filter are copied from the example in Fig. 10.

In Fig. 13a, gain error with standard deviation of 4 LSB results in the characteristic spur clusters. In Fig. 13b, the gain calibration has been applied resulting in around $4 \mathrm{~dB}$ reduction in spurious levels. Most likely, this result can be improved with further optimization, but it was not performed in this study.

Digital offset error calibration is not covered in this study. In [6], Law et al. proposed a simple solution of digitizing and subtracting the mean of each channel.

\section{ACKNOWLEDGEMENT}

This project was funded by Finnish Academy project 325928 and Academy of Finland 6Genesis Flagship under Grant 318927.

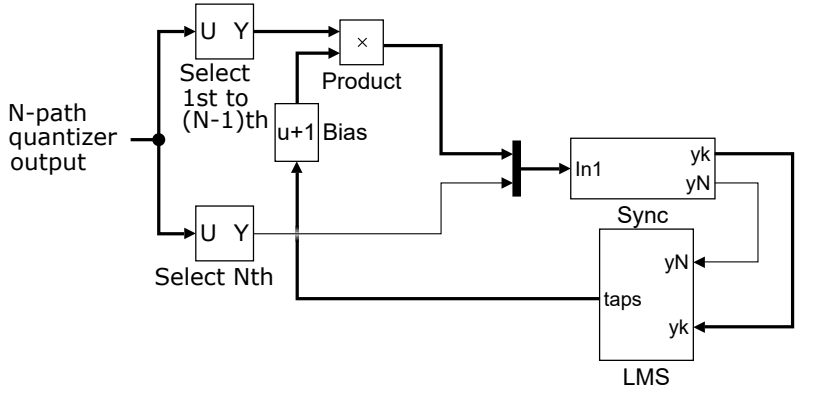

Fig. 12. Simulink model for the gain error calibration.

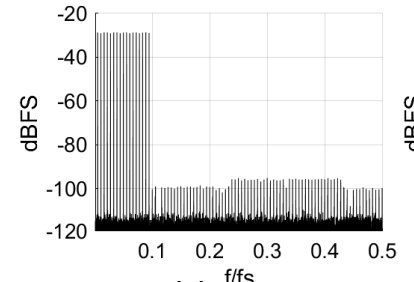

(a)

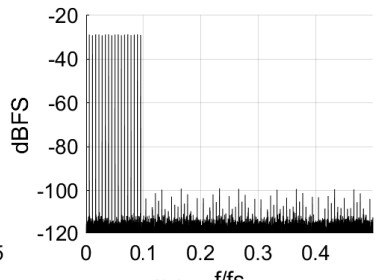

(b) $\mathrm{f} / \mathrm{fs}$

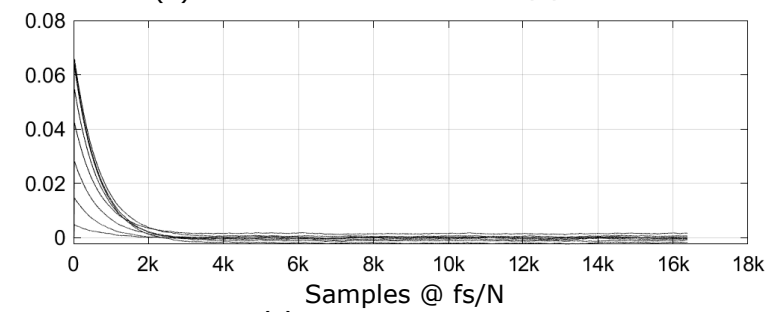

(c)

Fig. 13. 12-bit TI-ADC with 17-tone stimulus, $\{\mathrm{N}, \mathrm{OSR}\}=\{9,5 / 9\}$ and $\sigma=4$ LSB gain error: a) before gain error calibration, b) after gain error calibration and c) LMS tap convergence for the latter.

\section{CONCLUSION}

Fully digital timing error calibration scheme for timeinterleaved ADC was proposed. The proposed method is capable of compensating the path-related timing errors below Nyquist rate of the individual ADC. Moreover, the proposed method is applicable to any number of paths.

The proposed calibration structure involves both fixed and variable fractional delay filtering along with an adaptive LMS filtering. The aperture delay mismatch estimation by LMS involves filtering that makes calibration insensitive to offset error and gain error along with the imperfections of fractional delay filtering.

This work also presented a gain error calibration method that can be constructed with minor changes to the proposed timing error mismatch calibration structure.

For a given TI-ADC environment and stimulus, the timing mismatch calibration requires optimization that can be performed using a behavioral model. This work covered behavioral simulations with various number of paths and oversampling ratios, so the depth of optimization options was kept normative but minimal. Nevertheless, the calibration was found effective in all the test cases swept. 


\section{REFERENCES}

[1] B. Murmann, "Digitally assisted data converter design," 2013 Proceedings of the ESSCIRC (ESSCIRC), Bucharest, 2013, pp. 24-31, doi: 10.1109/ESSCIRC.2013.6649063.

[2] C. Vogel and H. Johansson, "Time-interleaved analog-to-digital converters: status and future directions," 2006 IEEE International Symposium on Circuits and Systems, Island of Kos, 2006, pp. 4 pp.-3389, doi: 10.1109/ISCAS.2006.1693352.

[3] S. M. Jamal, D. Fu, N. C. -J. Chang, P. J. Hurst and S. H. Lewis, "A 10-b 120-Msample/s time-interleaved analog-to-digital converter with digital background calibration," in IEEE Journal of Solid-State Circuits, vol. 37, no. 12, pp. 1618-1627, Dec. 2002, doi: 10.1109/JSSC.2002.804327.

[4] P. Satarzadeh, B. C. Levy and P. J. Hurst, "A parametric polyphase domain approach to blind calibration of timing mismatches for $\mathrm{M}$ channel time-interleaved ADCs," Proceedings of 2010 IEEE International Symposium on Circuits and Systems, Paris, 2010, pp. 4053-4056, doi: 10.1109/ISCAS.2010.5537632.

[5] J. Elbornsson, F. Gustafsson and J. -. Eklund, "Blind adaptive equalization of mismatch errors in a time-interleaved A/D converter system," in IEEE Transactions on Circuits and Systems I: Regular Papers, vol. 51, no. 1, pp. 151-158, Jan. 2004, doi: 10.1109/TCSI.2003.821300.

[6] C. H. Law, P. J. Hurst and S. H. Lewis, "A Four-Channel TimeInterleaved ADC With Digital Calibration of Interchannel Timing and Memory Errors," in IEEE Journal of Solid-State Circuits, vol. 45, no. 10, pp. 2091-2103, Oct. 2010, doi: 10.1109/JSSC.2010.2061630.

[7] N. Kurosawa, H. Kobayashi, K. Maruyama, H. Sugawara and K. Kobayashi, "Explicit analysis of channel mismatch effects in timeinterleaved ADC systems," in IEEE Transactions on Circuits and Systems I: Fundamental Theory and Applications, vol. 48, no. 3, pp. 261271, March 2001, doi: 10.1109/81.915383.

[8] T. I. Laakso, V. Välimaki, M. Karjalainen and U. K. Laine, "Splitting the unit delay [FIR/all pass filters design]," in IEEE Signal Processing Magazine, vol. 13, no. 1, pp. 30-60, Jan. 1996, doi: 10.1109/79.482137.

[9] B. Widrow et al., "Adaptive noise cancelling: Principles and applications," in Proceedings of the IEEE, vol. 63, no. 12, pp. 1692-1716, Dec. 1975, doi: 10.1109/PROC.1975.10036.

[10] S. Haykin "Adaptive Filter Theory," 3rd ed. Prentice Hall, 989 p, 1996.

[11] C. W. Farrow, "A continuously variable digital delay element," 1988. , IEEE International Symposium on Circuits and Systems, Espoo, Finland, 1988, pp. 2641-2645 vol.3, doi: 10.1109/ISCAS.1988.15483.

[12] A. Yardim, G. D. Cain and A. Lavergne, "Performance of fractionaldelay filters using optimal offset windows," 1997 IEEE International Conference on Acoustics, Speech, and Signal Processing, Munich, 1997, pp. 2233-2236 vol.3, doi: 10.1109/ICASSP.1997.599495.

[13] G. Oetken, "A new approach for the design of digital interpolating filters," in IEEE Transactions on Acoustics, Speech, and Signal Processing, vol. 27, no. 6, pp. 637-643, December 1979, doi: 10.1109/TASSP.1979.1163316.

[14] K. Pearson, "On the Criterion that a given System of Deviations from the Probable in the Case of a Correlated System of Variables is such that it can be reasonably supposed to have arisen from Random Sampling". Philosophical Magazine. Series 5. Vol. 50 no. 302. pp. 157-175, 1900. 